TITLE:

\title{
True molecular resolution in liquid by frequency-modulation atomic force microscopy
}

$\operatorname{AUTHOR}(\mathrm{S})$ :

Fukuma, T; Kobayashi, K; Matsushige, K; Yamada, H

CITATION:

Fukuma, T ... [et al]. True molecular resolution in liquid by frequency-modulation atomic force microscopy. APPLIED PHYSICS LETTERS 2005, 86(19): 193108.

\section{ISSUE DATE:}

2005-05-09

URL:

http://hdl.handle.net/2433/39672

\section{RIGHT:}

Copyright 2005 American Institute of Physics. This article may be downloaded for personal use only. Any other use requires prior permission of the author and the American Institute of Physics. 


\title{
True molecular resolution in liquid by frequency-modulation atomic force microscopy
}

\author{
Takeshi Fukuma \\ Department of Electronic Science and Engineering, Kyoto University, Katsura, Nishikyo, \\ Kyoto 615-8510, Japan \\ Kei Kobayashi \\ International Innovation Center, Kyoto University, Yoshida-Honmachi, Sakyo, Kyoto 606-8501, Japan
}

Kazumi Matsushige and Hirofumi Yamada ${ }^{\text {a) }}$

Department of Electronic Science and Engineering, Kyoto University, Katsura, Nishikyo, Kyoto 615-8510, Japan

(Received 13 December 2004; accepted 25 March 2005; published online 4 May 2005)

\begin{abstract}
The increasing attention directed towards nanobiological science requires high-resolution imaging tools for the liquid environment. We have been successful in recording molecular-resolution images of polydiacetylene in water with the frequency-modulation atomic force microscopy (FM-AFM). With the oscillation amplitude of a force-sensing cantilever reduced to $0.20 \mathrm{~nm}$, we were able to overcome the large frequency noise due to the low Q-factor of cantilever resonance in water. We have obtained vertical and lateral resolutions of $10 \mathrm{pm}$ and $250 \mathrm{pm}$, respectively. This method enables nondestructive imaging of soft biological samples with a load force on the order of $1 \mathrm{pN}$.

(C) 2005 American Institute of Physics. [DOI: 10.1063/1.1925780]
\end{abstract}

Submolecular-scale imaging in liquid is essential for understanding three-dimensional structures of biological materials, such as deoxyribonucleic acid and proteins in their physiological environments. Atomic force microscopy (AFM) (Ref. 1) has been the most promising candidate for this goal because with the ability to image a wide range of samples in various liquids and with subnanometer-scale resolution. However, molecular-resolution imaging in liquid by contact-mode AFM (CM-AFM) is possible only if the sample has a crystal-like structure or is firmly fixed on a substrate. ${ }^{2-4}$ Otherwise, the sample is damaged due to the large friction force acting between the tip and the sample. In dynamic-mode AFM, (DM-AFM), ${ }^{5}$ vertical motion of the cantilever significantly reduces the lateral friction force, ${ }^{6}$ and this allows us to image a weakly bound biological samples in liquid. ${ }^{7,8}$ However, the spatial resolution of DM-AFM in liquid has been no better than a few nanometers because of the extremely low $Q$ factor of the cantilever resonance. ${ }^{5,9}$ Here we present the result showing true molecular (i.e., subnanometer-scale) resolution of DM-AFM in liquid using frequency modulation (FM) detection method. ${ }^{10}$

In DM-AFM using FM detection method (FM-AFM: frequency-modulation AFM) ${ }^{10}$ a cantilever is used as a mechanical resonator in a self-oscillation circuit such that the cantilever vibrates at its resonance frequency with a constant oscillation amplitude $(A)$. The frequency shift $(\Delta f)$ of the cantilever resonance induced by the tip-sample interaction force is detected and used for the tip-sample distance regulation. Although a large number of molecular-scale images were acquired by FM-AFM, those images were taken only in ultrahigh-vacuum environments. The frequency noise in liquid is much larger than that in a vacuum due to the low $Q$ factor of the cantilever resonance. This leads to a low force sensitivity and a low spatial resolution in FM-AFM. In this

${ }^{\text {a)} E l e c t r o n i c ~ m a i l: ~ h-y a m a d a @ k u e e . k y o t o-u . a c . j p ~}$ study, we enhanced the sensitivity of the frequency signal to the short-range interaction forces by reducing the cantilever oscillation amplitude to the scale of the chemical bond length, namely, $0.2-0.3 \mathrm{~nm}^{11,12}$ In vacuum environments, small-amplitude operation requires high cantilever stiffness to avoid tip adhesion, ${ }^{11}$ which partially reduces the benefit of sensitivity enhancement. In contrast, due to the small longrange attractive interaction forces in liquid, ${ }^{13,14}$ smallamplitude FM-AFM without the tip adhesion is realized even with a relatively soft cantilever.

The FM-AFM apparatus used in this experiment was developed by modifying a commercially available AFM (JEOL: JSPM-4200). The AFM control electronics was replaced with other electronic circuits. ${ }^{15}$ The original cantilever deflection sensor was replaced with our homebuilt optical beam deflection sensor where the deflection noise density in liquid was about $40 \mathrm{fm} / \sqrt{\mathrm{Hz}}$. A highly doped $n$-Si cantilever (Nanosensors: $\mathrm{NCH}$ ) was used as the force sensor. The sample used here was a single crystal of polydiacetylene, 2,4-hexadine-1,6-diol bis( $p$-toluene sulfonate), which is hereafter referred to as "poly-PTS". A clean surface of the $b c$ plane of poly-PTS was prepared by cleaving it in air. Figures 1(a) and 1(b) show the crystal structures of the $b c$ plane and
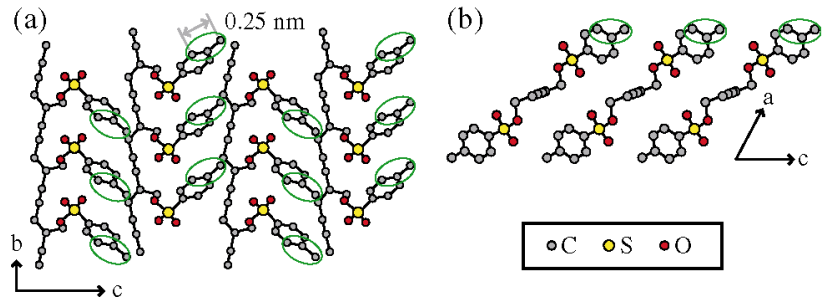

FIG. 1. (Color) Crystal structures of (a) $b c$ plane and (b) $a c$ plane of polyPTS. The lattice constants of the crystal are $a=1.493 \mathrm{~nm}, b=0.4910 \mathrm{~nm}$, and $c=1.4936 \mathrm{~nm}$. In the $b c$ plane, one side of the PTS side groups is located under the other side. Thus, they are omitted in (a) for clarity. Hydrogen atoms are also omitted to avoid complexity. 


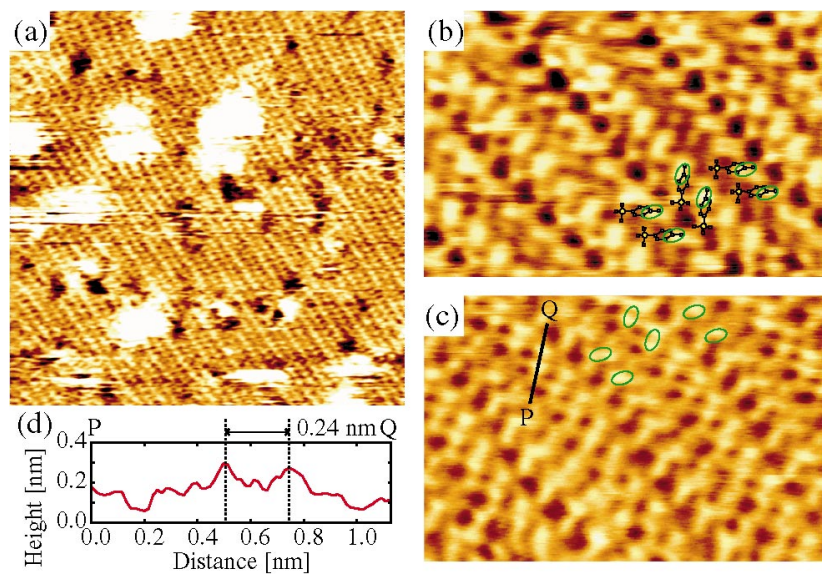

FIG. 2. (Color) FM-AFM images of the $b c$ plane of the poly-PTS single crystal taken in water $\left(Q=27, f_{0}=140 \mathrm{kHz}, k=42 \mathrm{~N} / \mathrm{m}\right)$. (a) $30 \mathrm{~nm} \times 30 \mathrm{~nm}, A=0.26 \mathrm{~nm}, \Delta f=+167 \mathrm{~Hz}$, scanning speed: $839 \mathrm{~nm} / \mathrm{s}$. (b) and (c) $6 \mathrm{~nm} \times 4 \mathrm{~nm}, A=0.20 \mathrm{~nm}, \Delta f=+290 \mathrm{~Hz}$, scanning speed: $448 \mathrm{~nm} / \mathrm{s}$. (d) Cross-sectional plot measured along the black line PQ indicated in (c).

$a c$ plane of poly-PTS. ${ }^{16}$ In the $b c$ plane, one side of the $p$-toluene sulfonate (PTS) side groups attached to the polydiacetylene main chain protrudes from the molecular $b c$ plane, while the other side of the PTS side groups and the main chains are located under the upper PTS side groups. Thus, the topmost carbon atoms indicated by the green circles should appear as bright protrusions in AFM images of the $b c$ plane. ${ }^{3}$

Figure 2(a) shows an FM-AFM image of the $b c$ plane of the poly-PTS single crystal taken in water. The image shows that the surface is composed of striped structures with a period of about $0.75 \mathrm{~nm}$, which agrees well with half the lattice constant along the $c$ axis. Each line consists of a series of small dots with a spacing of about $0.5 \mathrm{~nm}$, which corresponds to the unit cell length along the $b$ axis. Thus, individual PTS side groups are clearly resolved in the image. In addition, several dark spots corresponding to a number of structural defects in the polymer crystal are imaged with the molecular-scale features, demonstrating true molecular resolution of FM-AFM in water. The large bright areas are covered with contamination adsorbed on the surface.

By further reducing the oscillation amplitude to $0.20 \mathrm{~nm}$, we obtained FM-AFM images with improved spatial resolution. In Fig. 2(b), the large bright spots indicated by the green circles correspond to the topmost carbon atoms indicated by green circles in Fig. 1. In addition, some small bright spots are seen in Fig. 2(b). According to the molecular structure of the side chain, the small spots represent the sulfonate groups in the PTS side groups. With a better tip condition, the large bright spots are resolved into the two peaks, as shown in Fig. 2(c). The cross-sectional plot measured along the black line PQ [Fig. 2(d)] shows that the distance between the two peaks is about $0.24 \mathrm{~nm}$. As indicated in Fig. 1(a), the distance agrees with the distance between adjacent carbon atoms in the toluene group, about $0.25 \mathrm{~nm}$. The result demonstrates that FM-AFM in water can achieve a lateral resolution of $0.25 \mathrm{~nm}$.

Figure 3(a) shows the frequency shift-distance curve measured on the $b c$ plane of the poly-PTS single crystal in water. Figure 3(b) shows the force-distance curve derived from the results in Fig. 3(a) with the method proposed by Downloaded 30 May 2007 to 130.54 .110.22. Redistribution subject
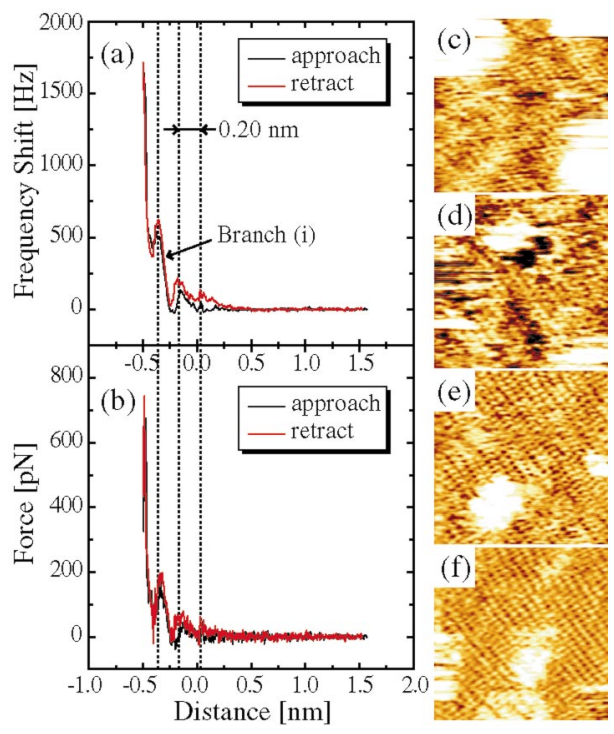

FIG. 3. (Color) (a) Frequency shift-distance curve measured on the $b c$ plane of the poly-PTS single crystal in water $\left(A=0.26 \mathrm{~nm}, Q=27, f_{0}\right.$ $=140 \mathrm{kHz}, k=42 \mathrm{~N} / \mathrm{m}$ ). (b) Force-distance curve derived from (a) using the method proposed by Giessibl (see Ref. 17). The black and red curves shown in (a) and (b) are measured in the approaching and retracting processes, respectively. Each curve was taken with a tip velocity of $0.7 \mathrm{~nm} / \mathrm{s}$ and approximately 1000 data points. (c)-(f) A series of FM-AFM images of the same sample taken with different frequency shifts $(15 \mathrm{~nm}$ $\left.\times 15 \mathrm{~nm}, A=0.26 \mathrm{~nm}, Q=27, f_{0}=140 \mathrm{kHz}, k=42 \mathrm{~N} / \mathrm{m}\right)$. The frequency shifts used for the imaging were (c) $+80 \mathrm{~Hz},(\mathrm{~d})+230 \mathrm{~Hz},(\mathrm{e})+385 \mathrm{~Hz}$, and (f) $+580 \mathrm{~Hz}$. Scanning speed was $839 \mathrm{~nm} / \mathrm{s}$. The images were obtained immediately before taking the curves shown in (a).

Giessibl. ${ }^{17}$ Figures 3(c)-3(f) show a series of FM-AFM images of the same sample taken with different frequency shifts. These images were obtained immediately before the measurement of the curves shown in Fig. 3(a). Individual PTS side groups are resolved in all the images, revealing that molecular resolution is stable in the frequency range from +80 to $+580 \mathrm{~Hz}$. This feature facilitates the selection of a setpoint for true molecular-resolution imaging.

The frequency shifts used for taking the FM-AFM images shown in Figs. 3(c)-3(f) were mostly in Branch (i) indicated in Fig. 3(a). The frequency-shift gradient of Branch (i) is $\partial(\Delta f) / \partial z=-2.1 \times 10^{13} \mathrm{~Hz} / \mathrm{m}$. On the other hand, the frequency noise density measured with a fast Fourier transform (FFT) analyzer shows a nearly constant value of 1.82 $\mathrm{Hz} / \sqrt{\mathrm{Hz}}$ at frequencies less than the FM bandwidth of $1 \mathrm{kHz}$. Thus, the frequency noise is $\delta f=57.6 \mathrm{~Hz}$ with an FM bandwidth of $1 \mathrm{kHz}$. Accordingly, the vertical resolution of FMAFM is $\delta z=\delta f /[\partial(\Delta f) / \partial z]=10 \mathrm{pm}$ at Branch (i). Since the vertical corrugation of the organic molecular surface is in the range of $20-100 \mathrm{pm},{ }^{18}$ vertical resolution of $10 \mathrm{pm}$ is sufficiently small to achieve true molecular resolution. The result indicates that the increase in frequency noise due to the decrease in the $Q$ factor in liquid was compensated for by the signal increase using small-amplitude operation in FM-AFM.

The force curve shown in Fig. 3(b) indicates the force oscillation that is caused by the layered structure of the water molecules confined in the tip-sample separation. ${ }^{19}$ The period of force oscillation is about $0.20 \mathrm{~nm}$, which roughly agrees with the previously reported values. ${ }^{19}$ The result reveals that such a small force oscillation with an amplitude of less than $100 \mathrm{pN}$ can be measured by FM-AFM, which indicates the excellent force sensitivity of FM-AFM in liquid to AIP license or copyright, see http://apl.aip.org/apl/copyright.jsp 
environments. The minimum detectable force $(\delta F)$ estimated by the small-amplitude approximation ${ }^{10}$ is given by $\delta F$ $=2 \mathrm{kA} \delta f / f_{0}=9.0 \mathrm{pN}$ at an FM bandwidth of $1 \mathrm{kHz}$. This load force is much smaller than the force used for the imaging of proteins in liquid by CM-AFM $(\sim 100 \mathrm{pN}){ }^{4}$ This indicates the ability of FM-AFM to image soft biological samples.

Since Figs. 3(c)-3(f) were obtained at Branch (i), the tip might be on one of the water layers at the closest tip position during the imaging. There is also a possibility that the tip might be covered with some water layers. A detailed understanding of the behavior of the water molecules during the high-resolution imaging and its contribution to the image contrast require further experiments and theoretical studies.

The use of true molecular-resolution imaging by smallamplitude FM-AFM in liquid opens a variety of applications in nanobiology and organic molecular science. These applications include not only structural imaging but also measurements of local electrical and magnetic properties. ${ }^{20,21}$ Force spectroscopy with small-amplitude FM-AFM in liquid provides quantitative information on the behavior of molecules in a liquid phase, as shown in Fig. 3(b). The spatial resolution and force sensitivity of this technique should be enhanced in the near future by using a smaller cantilever having a higher resonance frequency and a higher $Q$ factor in liquid.

The authers thank C. F. Quate for valuable comments and S. Okada and H. Nakanishi for providing poly-PTS single crystals. This work was supported by a Grant-in-Aid and a Leading Project on Nanotechnology and Materials from the Ministry of Education, Culture, Sports, Science, and Technology of Japan, and the 21st Century Center of Excellence Program, Kyoto University.
${ }^{1}$ G. Binnig, C. F. Quate, and C. Gerber, Phys. Rev. Lett. 56, 930 (1986).

${ }^{2}$ H. Yamada, S. Akamine, and C. F. Quate, Ultramicroscopy 42, 1044 (1992).

${ }^{3}$ H. Yamada, S. Okada, T. Fujii, M. Kageshima, A. Kawazu, H. Matsuda, H. Nakanishi, and K. Nakayama, Appl. Surf. Sci. 65, 366 (1993).

${ }^{4}$ D. J. Muller, F. A. Schabert, G. Buldt, and A. Engel, Biophys. J. 68, 1681 (1995).

${ }^{5}$ Y. Martin, C. C. Williams, and H. K. Wickramasinghe, J. Appl. Phys. 61, $4723(1987)$

${ }^{6}$ Q. Zhong, D. Inniss, K. Kjoller, and V. B. Elings, Surf. Sci. 290, L688 (1993).

${ }^{7}$ P. K. Hansma, J. P. Cleveland, M. Radmacher, D. A. Walters, P. E. Hillner, M. Bezanilla, M. Fritz, D. Vie, H. G. Hansma, C. B. Prater, J. Massie, L. Fukunaga, J. Gurley, and V. Elings, Appl. Phys. Lett. 64, 1738 (1994).

${ }^{8}$ C. A. J. Putman, K. O. V. der Werf, B. G. D. Grooth, N. F. V. Hulst, and J. Greve, Appl. Phys. Lett. 64, 2454 (1994).

${ }^{9}$ J. Tamayo, A. D. L. Humphris, and J. J. Miles, Appl. Phys. Lett. 77, 582 (2000).

${ }^{10}$ T. R. Albrecht, P. Grütter, D. Horne, and D. Rugar, J. Appl. Phys. 69, 668 (1991).

${ }^{11}$ F. J. Giessibl, H. Bielefeldt, S. Hembacher, and J. Mannhart, Appl. Surf. Sci. 140, 352 (1999).

${ }^{12}$ F. J. Giessibl, S. Hembacher, S. Bielefeldt, and J. Mannhart, Science 289, $422(2000)$

${ }^{13}$ U. Hartmann, Phys. Rev. B 43, 2404 (1991).

${ }^{14}$ B. Drake, C. B. Prater, A. L. Weisenhorn, S. A. Gould, T. R. Albrecht, C. F. Quate, D. S. Cannell, H. G. Hansma, and P. K. Hansma, Science 243, 1586 (1989).

${ }^{15}$ K. Kobayashi, H. Yamada, H. Itoh, T. Horiuchi, and K. Matsushige, Rev. Sci. Instrum. 72, 4383 (2001)

${ }^{16}$ V. D. Kobelt and E. F. Paulus, Acta Crystallogr., Sect. B: Struct. Crystallogr. Cryst. Chem. 30, 232 (1974).

${ }^{17}$ F. J. Giessibl, Appl. Phys. Lett. 78, 123 (2001).

${ }^{18} \mathrm{H}$. Yamada, Noncontact Atomic Force Microscopy (Springer-Berlin, 2002), Chap. 12.

${ }^{19}$ J. N. Israelachvili and R. M. Pashley, Nature (London) 306, 249 (1983).

${ }^{20}$ S. Kitamura and M. Iwatsuki, Appl. Phys. Lett. 72, 3154 (1998).

${ }^{21}$ T. R. Albrecht, P. Grütter, D. Rugar, and D. P. E. Smith, Ultramicroscopy 42, 1638 (1992). 